\title{
Morphology and optical constants of nanographite films created by thermal vacuum deposition
}

\author{
V.V. Kozachenko ${ }^{1}$, O.S. Kondratenko ${ }^{2}$, F.Le Normand ${ }^{3}$ \\ ${ }^{I}$ Taras Shevchenko Kyiv National University, Department of Physics \\ 64, Volodymyrska str., 01601 Kyiv, Ukraine \\ ${ }^{2} V$. Lashkaryov Institute of Semiconductors Physics, NAS of Ukraine, \\ 45, prospect Nauky, 03028 Kyiv, Ukraine \\ ${ }^{3}$ Institut d'Electronique du Solide et des Systèmes (InESS), \\ 23 rue du Loess, 67037 Strasbourg Cedex 2, France
}

\begin{abstract}
The method for nanographite films preparation using thermal sublimation in vacuum of graphite nanoparticles under ultrasound treatment was proposed. Studied in the system nanographite film - substrate were composition and morphology of a multilayer interface system by using ellipsometry and Raman scattering. It has been ascertained that the optical parameters of discontinuous nanographite films strongly depend on morphology of the surface layer. Using the results of ellipsometric measurements, we determined the effective optical constants, thickness of the films and calculated the volume composition of the solid phase in the film within the framework of the model of effective environment by Bruggeman.
\end{abstract}

Keywords: nanographite films, ultrasound, optical constants, ellipsometry, Raman.

Manuscript received 15.10.14; revised version received 22.12.14; accepted for publication 19.02.15; published online 26.02.15.

\section{Introduction}

Recently, attention of scientists was attracted by the graphene - graphite monoatomic layer due to its properties, namely: high transparency in a wide spectral range, electric conductivity and durability characteristics, which makes it a promising element in electronics [1]. However, the lack of graphene band gap leads to difficulties in creation of the field-effect transistor. One solution of this problem is to create graphene nanotape, where quantum size effects allow getting the band gap of the required width [2]. Some interest is also caused by another form of carbon graphite nanocrystals composed of a few to several tens of parallel flat graphene layers [3]. Unlike other carbon nanomaterials, nanographite can be prepared much easier. Particularity of nanoscale materials is that their properties are very dependent on their shape and size, which creates a wide field for researches.

In this study, the new method for preparation of nanographite films by using the thermal vacuum deposition process was proposed. As demonstrated experimentally [4], it is possible to realize nanographite powder synthesis in various solvents under powerful ultrasound (US) treatment. Researches of this nanographite showed that the action of ultrasound (frequency of $20.4 \mathrm{kHz}$, power density of $0.1 \ldots 1 \mathrm{~W} / \mathrm{cm}^{3}$ ) for $5 \ldots 10 \mathrm{~min}$ forces the graphite to transform to 
nanographite particles with the lateral sizes $300 \ldots 500 \mathrm{~nm}$ and thickness of 30 up to 50 graphene layers. Optical methods are used to determine the geometrical parameters of heterosystem and optical constants of their components. Since the optical parameters determine efficiency (sensitivity) of certain devices, determination of these parameters and ways of their modification is a problem that needs to be solved.

\section{Experimental techniques}

In our work, we prepared nanographite by dispersing graphite powder (average particle size of $75 \mu \mathrm{m}$ ) in acetone by entering ultrasound with the specific power close to $2 \mathrm{~W} / \mathrm{cm}^{2}$ at the frequency $40 \mathrm{kHz}$. Graphite was crushed in the ultrasonic bath for 10 hours. We used a much longer time for exposing the graphite to US, higher frequency and higher specific power of US. After treatment and evaporation of the solvent, we obtained superdispersed nanographite, powder substance was dark grey. The size of graphite nanoparticles was tens of nanometers.

Based on the presence of nanosized particles in graphite powder obtained, it was suggested that they can sublimate when heated. For sublimation of atoms and whole clusters of matter, the method of thermal deposition in vacuum was used. The temperature of evaporator with nanographite powder was $1000^{\circ} \mathrm{C}$. It was determined by the pyrometric temperature measurement method for luminance of evaporator and strength of the current passing through it. The pressure in the vacuum chamber was about $10^{-5}$ Torr, substrate temperature during deposition remained equal to $20^{\circ} \mathrm{C}$. As substrates, glass plates with the thickness of $500 \mu \mathrm{m}$ were used.

Optical properties of the samples were studied using the ellipsometry methods and Raman scattering. Ellipsometric measurements were carried out on a monochromatic ellipsometer LEF-3M with working wavelength $\lambda=632.8 \mathrm{~nm}$ and within the working range of light incidence angles from 45 to 90 degrees.

Surface morphology of nanographite film was studied using atomic force microscopy (AFM). In this paper, for studying the surface of nanographite film, the AFM of type NT-MDT NTEGRA Prima Scanning Probe Microscope was used. Measurements were carried out in air at room temperature using the silicon nitride tip on the elastic console element with the stiffness ratio $0.01 \ldots 0.6 \mathrm{~N} / \mathrm{m}$ in the semi-contact mode.

\section{Results and discussion}

Thermogravimetric (TG) study of nanographite powder shows high temperature stability of nanographite particles in air up to the temperature $600{ }^{\circ} \mathrm{C}$, above which active carbon oxidation takes place. The minimum derivative of the weight loss (the most intense carbon oxidation) is observed at the temperature $750{ }^{\circ} \mathrm{C}$ (Fig. 1). For temperatures higher than $850{ }^{\circ} \mathrm{C}$, the residual weight was smaller and limited to the error in weight not exceeding $0.25 \%$, which indicates absence of impurities. Besides, in the $200{ }^{\circ} \mathrm{C}$ temperature region, the derivative of the weight loss reaches its peak, which is associated with active evaporation of moisture, carbon monoxide and carbon dioxide adsorbed onto the powder surface developed. Their total concentration was about 5\% (wt.).

To obtain nanographite film by thermal evaporation of material in vacuum, we precipitated graphite nanoparticles on glass substrates. Our assumption was based on the fact that the temperature of the evaporator is not enough [5] for sublimation of graphite single atoms and, therefore, only integer quantity of nanographite particles can be settled on a substrate. It is obvious that, in nanographite powder obtained, there were significantly large variations in particle size, and in the process of thermal deposition onto a substrate, the probability for the smallest particles to get on a substrate was the highest one.

The resulting film was semitransparent, of yellowish color. It may indicate to a change of optical properties of nanocrystalline graphite as compared to the bulk material.

One of the most effective optical methods of researching the properties of the interface of two media and thin-film heterostructures is ellipsometry, when two quantitative characteristics (amplitude ratio $\Psi$ and phase difference $\Delta$ ) of polarized light reflected from the surface are examined simultaneously, which allows to determine both the thickness and optical constants of layers. This change in optical constants of layers serves as the characteristic that contains information about microstructure, composition of multicomponent layers and porosity.

According to the ellipsometric measurements of the polarization angles $\Psi(\varphi)$ and $\Delta(\varphi)$, the optical constants are determined: refraction index $n$, absorption coefficient $k$ and thickness $d$ of nanographite film by solving the inverse ellipsometric task using the method of minimizing the quadratic objective function [6]. The one-layer model was used to calculate the optical

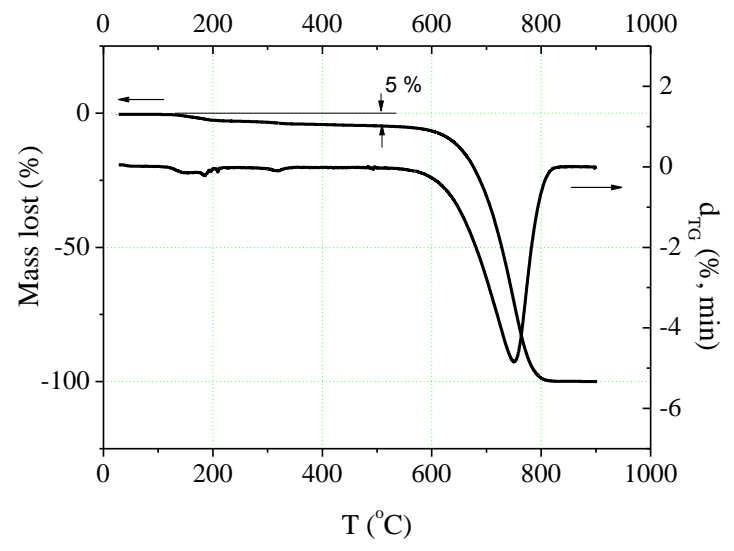

Fig. 1. TG results for nanographite powder. 


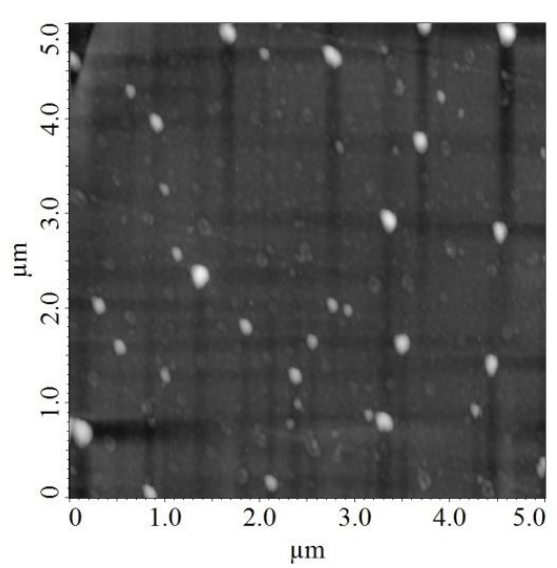

Fig. 2. AFM image of nanographite film.

parameters of the structure: effective composite layer of nanographite film consisting of nanoparticles of graphite and air on the semi-infinite substrate (glass). The obtained optical constants of the effective nanographite film are $N=1.69-i 0.69$. Because of the different structure and other defects, optical constants of semitransparent films are different from those for bulk materials. It turned out that the complex refractive index of nanographite layer at the optical wavelength $\lambda=$ $632.8 \mathrm{~nm}$ was less than the corresponding bulk material parameter (for example, $[7,8]$ ) due to porosity of the composite layer of material.

Using optical parameters of the structure, determined by the multi-angle ellipsometry method, the fraction of solid phase $f$ of nanographite layer was determined. To reach it, the model of effective (statistical) composite medium by Bruggeman was applied [9]. Modeling the system within a twocomponent model for effective film "nanoparticles of graphite and air (cavity)" on the substrate surface allowed us to define the parameters of the system. The parameters obtained for graphite: $d_{\mathrm{opt}}=30.00 \mathrm{~nm}, f_{\mathrm{graph}}=$ $46.2 \%, f_{\text {air }}=53.8 \%$.

These optical studies are in good correlation with the studies of surface morphology of nanographite film through the atomic force microscope investigation (AFM). The picture of the nanographite film surface obtained from the AFM measurements is shown in Fig. 2.

Analysis of the results of these studies shows that the graphite film in its structure is not solid but has an island nature. Using this method for obtaining nanographite film, grains of different sizes in height and diameter are formed on the substrate. So, we can see some large grains with the average height $80 \ldots 100 \mathrm{~nm}$, but their concentration is lower as compared to the main area of the sample. Basically, the film consists of grains, the average diameter of which is within the range $80 \mathrm{~nm}$; the average height of individual nanoparticles reaches approximately $25 \ldots 30 \mathrm{~nm}$.

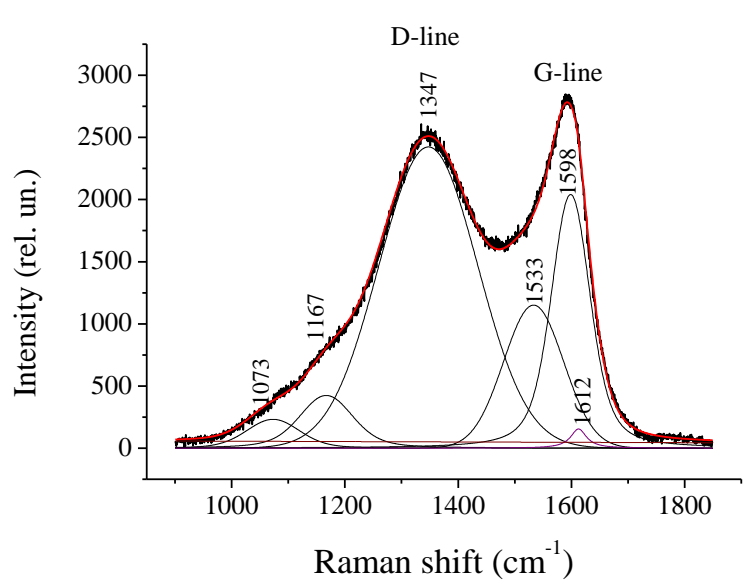

Fig. 3. Raman spectrum of nanographite film.

To ensure that our film is actually composed of nanographite particles, the Raman scattering spectrum was measured (Fig. 3). In the high-frequency region of the spectrum, two typical bands of the Raman spectrum of the first order, the so-called D- and G-bands ( 1350 and $\sim 1590 \mathrm{~cm}^{-1}, \quad$ respectively) are observed. Decomposition of the Raman spectra of the first order gave 6 peaks located at 1073, 1168, 1347 (D-line), 1533, 1598 (G-line) and $1612 \mathrm{~cm}^{-1}$ (D'-line). From [10], we know what can be responsible for these D-, G- and D'lines. G-band corresponds to $E_{2 g_{2}}$ vibration that is a sign of ordered graphite. D- and D'-lines describe the double resonance. Their appearance is related with the presence of defects at the edges and inside the graphene planes. The presence of this band indicates the availability of particles in the nanographite film under study [11]. The peak at $1168 \mathrm{~cm}^{-1}$ can be attributed to the local vibration mode at the boundary [12]. The peak at $1530 \mathrm{~cm}^{-1}$ reflects the presence of disorder in the structure in the form of amorphous carbon, which exists in the form of both intermediate defects outside the plane of the aromatic rings of $\mathrm{sp}^{3}$ bonds [11] and rings with an odd number of carbon atoms.

Using the Raman spectrum, the average lateral size of the nanocrystals contributing to the D-line can be calculated. The average lateral size $L_{a}$ is inversely proportional to the relative intensities of the D-line to $\mathrm{G}$ line. The proportionality coefficient is an empirical value [12], and for $L_{a}$ the formula can be written as follows:

$$
L_{a}(\mathrm{~nm})=\frac{560}{E_{l}^{4}} \cdot \frac{I_{G}}{I_{D}},
$$

where $E_{l}$ is the laser energy used to excite transitions in the Raman spectrum. If, instead of the energy, one uses the respective laser wavelength, the formula (1) can be written as

$$
L_{a}(\mathrm{~nm})=\left(2.4 \cdot 10^{-10}\right) \lambda_{l}^{4}\left(\frac{I_{G}}{I_{D}}\right) \text {. }
$$


In this case, the ratio of the intensities of the G- and D-lines was $I_{G} / I_{D}=1.13$. Having substituted the laser wavelength $\lambda_{l}=532 \mathrm{~nm}$, we can estimate the average lateral size of nanographite crystallites. It was equal approximately to $40 \mathrm{~nm}$, which correlates with the results of atomic force microscopy.

\section{Conclusions}

Thus, we have proposed the method for creating films with 2-D array graphite nanoparticles by thermal sublimation of graphite particles with average lateral size of $40 \mathrm{~nm}$ in vacuum $10^{-5}$ Torr on the surface of glass substrates, the graphite particles being preliminary dispersed using ultrasound treatment. The Raman scattering and AFM technique were used to show morphology of films with graphite nanoparticles.

The ellipsometric measurements have proved that optical parameters of discontinuous nanographite films strongly depend on morphology of the surface layer. Effective optical constants of the film change depending on the modification type of surface. It has been shown that using the results of ellipsometric measurements makes it possible to calculate not only the optical constants and thickness of the film but also to determine the volume composition $f$ of the solid phase in the film within the framework of the model of effective medium by Bruggeman.

\section{References}

1. P.J.F. Harris, Carbon Nanotubes and Related Structures: New Materials for the Twenty-First Century. Cambridge Univ. Press, Cambridge, 1999.

2. Z. Chen, Y.-M. Lin, M.J. Rooks, Ph. Avouris, Graphene nano-ribbon electronics // Physica E: Low-dimens. Systems and Nanostruct. 40, p. 228232 (2007).
3. V.G. Lytovchenko, M.V. Strikha, M.I. Klyui, Modified graphene-like films as a new glass of semiconductors with a variable energy GaP // Ukr. J. Phys. 56, p. 175-178 (2011).

4. Yu.V. Ioni, S.V. Tkachev, N.A. Bulychev, S.P. Gubin, Superdispersed nanographite // Inorganic Materials, 47, p. 671-677 (2011).

5. Yu.M. Lahtin, V.P. Leont'eva, Materials Technology, Handbook. Moscow, Manufacturing Publ., 1990 (in Russian).

6. V.N. Antonyuk, N.L. Dmitruk, M.F. Medvedeva, Ellipsometry in Science and Engineering, Handbook. Novosibirsk, Nauka Publ., 1987 (in Russian).

7. J. Weber, V. Calado, and M. Van de Sanden. Optical constants of graphene measured by spectroscopic ellipsometry // Appl. Phys. Lett. 97, 091904 (2010).

8. A. Matkovič, U. Ralevič, G. Isič et al., Spectroscopic ellipsometry and the Fano resonance modeling of graphene optical parameters // Phys. Scr. 149, 014069 (2012).

9. C.F. Bohren, D.R. Huffman, Absorption and Scattering of Light by Small Particles. Wiley, New York, 1983.

10. M.A. Pimenta, G. Dresselhaus, M.S. Dresselhaus et al., Studying disorder in graphite-based systems by Raman spectroscopy // Phys. Chem. Chem. Phys. 9, p. 1276 (2007).

11. V.A. Ermakov, Investigation of structural and chemical parameters of thin films, nanoglassceramics and multilayered nanographite by using Raman spectroscopy. $\mathrm{PhD}$ Thesis (Physics and Mathematics: 01.04.05), Sankt-Petersburg, 2011.

12. L.G. Cançado, K. Takai, T. Enoki et al., General equation for the determination of the crystallite size of nanographite by Raman spectroscopy // Appl. Phys. Lett. 88, p. 3 (2006). 